\title{
Simple Small-Signal HEMT Model Suitable for GaN Stability Analysis and Technologies Benchmarking
}

\author{
Ammar Issaoun*, Thomas Roedle \\ GaN Front-End Technology, Ampleon, Nijmegen, The Netherlands \\ Email address: \\ ammar.issaoun@ampleon.com (A. Issaoun), thomas.roedle@ampleon.com (T. Roedle) \\ ${ }^{*}$ Corresponding author
}

\section{To cite this article:}

Ammar Issaoun, Thomas Roedle. Simple Small-Signal HEMT Model Suitable for GaN Stability Analysis and Technologies Benchmarking. World Journal of Applied Physics. Vol. 6, No. 1, 2021, pp. 1-8. doi: 10.11648/j.wjap.20210601.11

Received: February 11, 2021; Accepted: February 20, 2021; Published: March 3, 2021

\begin{abstract}
This article deals with the extension of the small-signal model usage to GaN technologies benchmarking, and to the detection of internal oscillations occurring in highly optimized multi-finger GaN high-electron-mobility transistors (HEMTs). The proposed small-signal model consists of only 14 circuit elements. Its simple semi-analytical extraction procedure is developed in Keysight ADS circuit simulator, letting instantaneous comparison between modelled and simulated small-signal parameters. The simplicity and the adaptability of the technique always ensures a physical model parameter extraction. The technique is demonstrated for various technology processes, layouts, dimensions, and for three commercially available GaN vendors. The extracted data and the number of circuit elements are used to benchmark GaN technologies in terms of bias dependency, efficiency, and static linearity. By coupling the small-signal model to the electromagnetic (EM) GaN HEMT layout simulation results in a powerful tool for detecting odd-mode and even-mode instabilities. The technique is proven for various GaN basic cells as well as for power bars. Even prior to structure fabrication, the tool can be used to analyze its stability behavior by exploring its layout.
\end{abstract}

Keywords: HEMT, Small-Signal, Direct-Extraction, Stability, Odd-Modes, Even-Modes, Power Amplifiers, Benchmark

\section{Introduction}

The small-signal model is of great importance for technology process, and for device and application developers. In recent years, many researchers have been focused on designing application circuits using the emerging GaN technology, to make use of its high-power density, high breakdown field and high efficiency in compression. Small-signal modelling of GaN-based High-electron-mobility transistors (HEMTs) is becoming a hot research topic [1-9] in device modelling. A GaN HEMT small-signal model describes the device small-signal behavior. Hence, it's a cornerstone in device and in circuit designs, or in circuit stability analysis [6,7], and in large-signal modelling [8].

Scanning and transmission electron microscopes (SEM, TEM) used traditionally in failure analysis can be used to reveal process and layout differences between processes and/or technologies. The end users compare technologies at application level, in search of optimum efficiency and high linearity. GaN developers are interested in ways to connect application electrical requirements to technology at the early stage of process development. This is not a trivial task. Maybe, the best link between technology and its applications is a small-signal model. Hence, developing useful, practical, and reliable small-signal models along with their extraction methods are of great importance for GaN HEMT designers. Many papers are published on the two main small-signal modelling approaches equivalent circuit models and application of the intelligent modelling techniques. A comparison between the two approaches is presented in [1]. The standard equivalent circuit techniques show lower time consumption and complexity in development. They are based on direct optimization and/or hybrid approaches to get reliable parameter extraction [2-5]. Nevertheless, the robustness and the reliability of the small-signal extraction procedure depends on the measurement conditions and on the equivalent circuit used. A change in one of these two conditions will imply to rework the optimization procedure. A GaN HEMT small-signal model will depend on device layout, technology process, and substrate. Developing a 
generalized robust and reliable small-signal extraction procedure is not a simple task. GaN HEMT small-signal model users are in a search of simple and flexible way to build models in circuit simulators such as Keysight ADS. The 22-element GaN HEMT small-signal model developed in [10] continuously sees improvements i.e. [2-5].

In our study, an attempt was made to extract the 22-element model using genetic algorithm and most element values turned to be unphysical or have negative values. Hence, the present proposal is developed keeping the need of simplicity and of the practicability without losing accuracy. The technique consists of a simplified 14-element small-signal model that can expand depending on technology modelling needs. Its semi-analytical extraction procedure is developed in Keysight ADS circuit-simulator, allowing user to directly compare the modelled scattering, admittance, and impedance parameters to measurements. The obtained data was used to benchmark $\mathrm{GaN}$ technologies in terms of application parameters such as linearity, gain and efficiency.

Oscillations can occur in microwave power amplifiers at three levels DC, small-signal and large-signal. These oscillation regimes are also referred as odd-mode, even-mode, and parametric, respectively. Odd-mode oscillations happen when transistors are put in parallel while the impedances seen from their input/output are not identical. These oscillations are very critical in structures using transmission lines due to coupling between them. Even-mode oscillations occur when the connected load or source impedances provide a reflection coefficient magnitude bigger than the one at the input/output of the transistor. Large-signal oscillations may occur when a power amplifier operation is pushed to the nonlinear regime, due to variations of its nonlinear internal elements. The oscillation issue gives a lot of headaches for both circuit designers and technology developers. This stability issue can be investigated analytical by following two steps approach: model formulation, and detection. The outcome of a stability detection technique depends strongly on the accuracy of its input transfer function. Hence, stability problem analysis is directly related to the accuracy of the model used to describe the structure or the circuit behavior in both amplitude and phase. Circuit designers rely mostly on delivered foundry models to tackle the stability issue in their designs. For technology developers, such models are not available. Hence, developing tools to analyze and predict stability issue is of a considerable importance for them. The simplicity and the practicability of the tools are of equal importance.

In early process phases, probably the only link available between technology and mathematical stability formalism is the small-signal model. The simple and yet accurate approach described here, when coupled to an electromagnetic (EM) simulation of HEMT basic cells or power bars, can be used in the analysis of odd-mode and even-mode stability issue. The outline of the stability analysis method for a basic HEMT cell was presented in [7], without any detail about the associated small-signal approach neither about the EM simulation settings. Here, both EM and small-signal models are detailed, and the stability technique is further demonstrated for a complex power bar structure.

\section{Small-signal Model}

\subsection{Device Structure}

Figure 1 shows an example of a basic GaN HEMT cell, which consists of Ground-Signal-Ground (GSG) pads and a structure having one or multiple-fingers. In most cases, GSG pads are made identical for wafer area and modelling optimizations. The structure layout depends on design parameters such as gain, efficiency, peak power, and thermal behavior. A schematic cross-section through source-gate-drain area of a single finger structure is shown in Figure 2a. Its layout in a symbolic $3 \mathrm{D}$ representation is given in Figure $2 \mathrm{~b}$. The electrical equivalent circuit elements representing the different physics phenomena is also given in Figure $2 \mathrm{~b}$. The equivalent circuit is exploded in Figure 3 showing its 14-elements. All capacitances consist of two parts: metallic part coming from source, gate and drain interconnects and field plates and a semiconductor part, consisting of charges involved in the structure under the considered region.

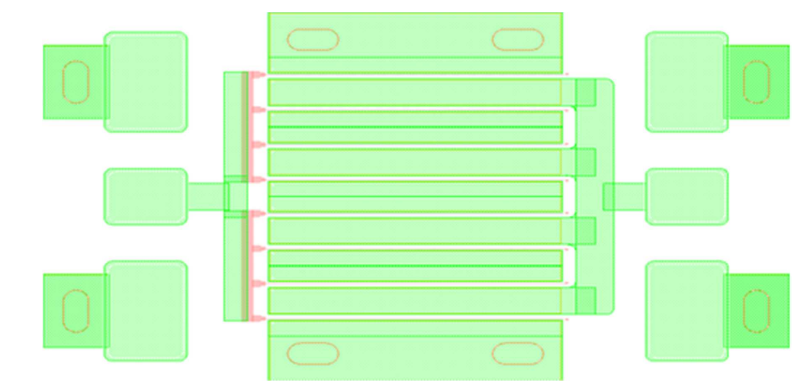

Figure 1. GSG basic GaN HEMT cell with 8 fingers.

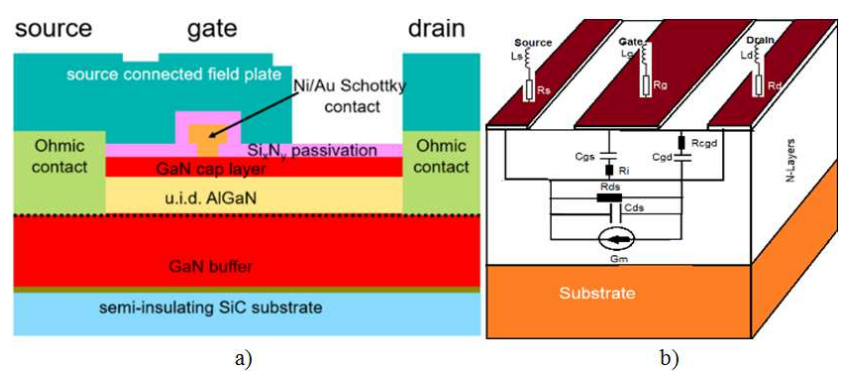

Figure 2. Single finger basic GaN HEMT cell; a) schematic cross-section; b) $3 D$ structure with corresponding electric parameters.

\subsection{Extraction Procedure}

The simple analytical equations developed in [11] are used to model the intrinsic or core part of the equivalent circuit of Figure 3. For convenience, they are repeated here:

$$
\begin{array}{r}
G_{m}=\operatorname{Re}\left[Y_{21}\right]_{w^{2}=0} \\
G_{d s}=\operatorname{Re}\left[Y_{22}\right]_{w^{2}=0} \\
R_{g}=\operatorname{Re}\left[Y_{11}\right] / \operatorname{Im}\left[Y_{11}\right]^{2}
\end{array}
$$




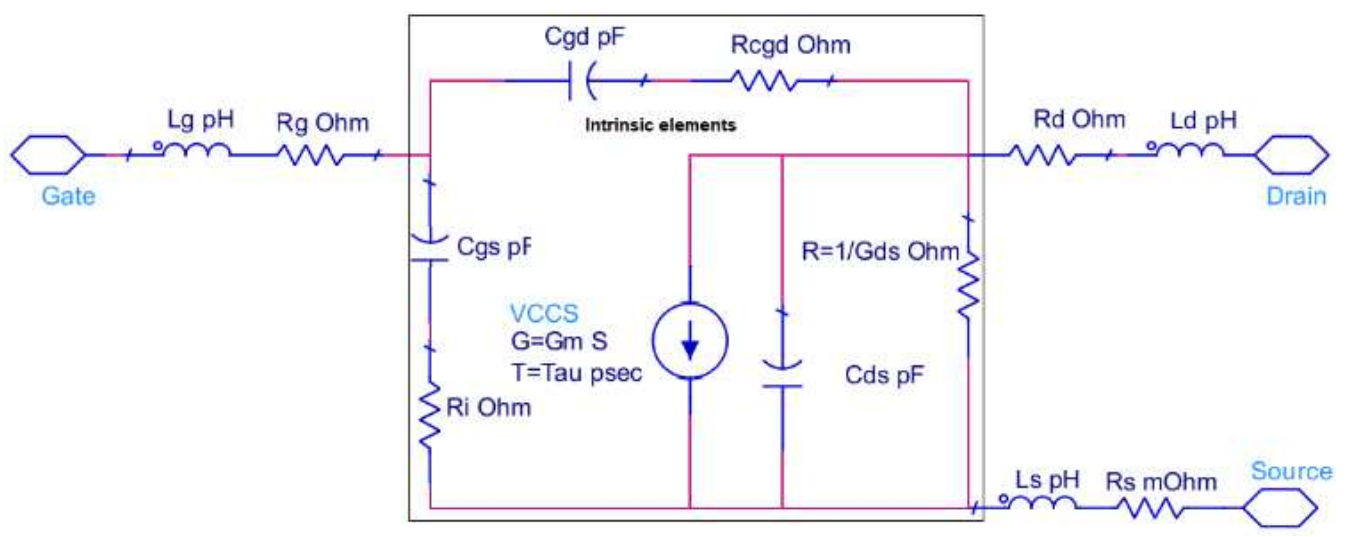

Figure 3. 14-elements small-signal distributed model for active GaN HEMT.

$$
\begin{gathered}
C_{d s}=\left(\operatorname{Im}\left[Y_{22}\right]+\operatorname{Im}\left[Y_{12}\right]\right) / w \\
C_{g s}=\left(\operatorname{Im}\left[Y_{11}\right]+\operatorname{Im}\left[Y_{12}\right]\right) / w \\
C_{g d}=-\operatorname{Im}\left[Y_{12}\right] / w
\end{gathered}
$$

In the above equation $\mathrm{w}$ stands for angular frequency. The extraction procedure steps are:

1. Evaluation of the above 6 equations in the simulator using measured scattering parameters of the structure under test,

2. Feeding the extracted values for $\mathrm{Cgs}, \mathrm{Cgd}, \mathrm{Cds}, \mathrm{Gm}, \mathrm{Gds}$ and $\mathrm{Rg}$ into the small-signal equivalent circuit. $\mathrm{Cgs}, \mathrm{Cgd}$, $\mathrm{Cds}, \mathrm{Gm}$ and Gds elements will not change for the rest of the procedure. Rg and Gds values might change due to their coupling effect with Ri and Rd, respectively,

3. Tuning of $\mathrm{Lg}$ and $\mathrm{Ld}$ inductors to the exact fit of S11 and S22 in Smith chart configuration,

4. Tuning of Ri to fit $\mathrm{dB}$ (S11) in the high GHz region. Ri value will impact the extracted low frequency $\mathrm{Rg}$ value. Hence, Rg and Ri must fit dB (S11) over frequency,

5. Tuning of Rd value to fit $\mathrm{dB}$ (S22) parameter in the high $\mathrm{GHz}$ region,

6. Tuning of Ls, Rs and Rcgd values to fit S21 parameter,

7. Tuning of Tau to fit phase (S21) at high frequency.

$\mathrm{Rg}, \mathrm{Lg}, \mathrm{Ld}, \mathrm{Rd}, \mathrm{Ls}$, and Rs values are independent with bias. It is recommended to verify their extraction in off- and on-states, to keep consistency of the circuit elements. It is also recommended to check the admittance $(\mathrm{Y})$ parameters fit.

\subsection{Extraction Procedure}

Firstly, all the studied devices are $\mathrm{AlGaN} / \mathrm{GaN}$ HEMTs with Silicon Carbide substrate (SiC) GSG mounted on a flange. All the measurements have been carried out with the AMCAD PIV3200 system at ambient temperature. The gate and drain (Vgsq, Vdsq) quiescent biasing for all the $\mathrm{S}$ parameters is $(-5 \mathrm{~V}$, $50 \mathrm{~V})$. The pulse signal has a pulse width of $1 \mu \mathrm{s}$ and $1 \mathrm{~ms}$ period.

Secondly, the above outlined procedure was applied for modelling a structure of $4 \times 400 \mu \mathrm{m}$ fabricated using a $0.25 \mu \mathrm{m}$ $\mathrm{GaN}$ process. Figure 4 compares the modelled versus measured scattering parameters of the structure. Apart from the achieved very good agreement between measured and simulated data; the figure shows the regions associated to each parameter extraction and/or verification. It is important to show the scattering parameters as given in Figure 4, for accurate and consistent extraction procedure.

Thirdly, the extraction procedure is further validated over structure layouts, dimensions, and technology process, as shown in Tables 1 and 2. Circuit elements not given in Tables 1 and 2 have zero as their values.

Table 1. Extracted small-signal parameters over bias conditions for $4 x 400 \mu \mathrm{m}$ structure based on $0.5 \mu \mathrm{m}$ GaN technology.

\begin{tabular}{lllllll}
\hline Id (mA) & $\mathbf{1 2}$ & $\mathbf{8 5}$ & $\mathbf{1 5 5}$ & $\mathbf{1 8 5}$ & $\mathbf{3 0 4}$ & $\mathbf{3 9 6}$ \\
\hline $\mathrm{Cds}(\mathrm{pF})$ & 0.81 & 0.80 & 0.80 & 0.77 & 0.77 & 0.75 \\
$\mathrm{Cgs}(\mathrm{pF})$ & 2.72 & 3.50 & 3.74 & 3.89 & 3.91 & 3.90 \\
$\mathrm{Cgd}(\mathrm{fF})$ & 75 & 64 & 59 & 55 & 54 & 54 \\
$\mathrm{Gm}(\mathrm{S})$ & 0.14 & 0.34 & 0.39 & 0.43 & 0.43 & 0.43 \\
Gds (mS) & 1.31 & 3.16 & 3.62 & 3.70 & 3.83 & 3.63 \\
Tau (ps) & 6 & 6 & 6 & 6 & 6 & 6 \\
$\operatorname{Rg}(\mathrm{W})$ & 2.4 & 2.4 & 2.4 & 2.4 & 2.4 & 2.4 \\
$\mathrm{Rd}(\mathrm{W})$ & 0.9 & 1.11 & 1.2 & 1.2 & 1.3 & 1.3 \\
$\mathrm{Ld}(\mathrm{pH})$ & 32 & 32 & 32 & 32 & 32 & 32 \\
$\mathrm{Lg}(\mathrm{pH})$ & 48 & 48 & 48 & 48 & 48 & 48 \\
\hline
\end{tabular}

Table 2. Extracted small-signal parameters for various structure geometries based on $0.5 \mu \mathrm{m}$ and $0.25 \mu \mathrm{m}$ GaN technologies.

\begin{tabular}{lllllll}
\hline Structures & \multicolumn{2}{l}{$\mathbf{2 x 4 0 0} \mathbf{u m}$} & \multicolumn{2}{l}{$\mathbf{4 x 3 0 0} \mathbf{~ u m}$} & \multicolumn{2}{l}{$\mathbf{4 x 4 0 0}(\mathbf{0 . 2 5})$} \\
\hline Id (mA) & OFF & $\mathbf{1 2}$ & OFF & $\mathbf{1 2}$ & OFF & $\mathbf{3 4}$ \\
\hline Cds (pF) & 0.40 & 0.41 & 0.51 & 0.46 & 0.53 & 0.54 \\
Cgs (pF) & 0.81 & 1.54 & 1.22 & 2.05 & 1.23 & 1.94 \\
Cgd (fF) & 42 & 34 & 61 & 44 & 99 & 86 \\
Gm (S) & 0 & 0.11 & 0 & 0.11 & 0 & 0.24 \\
Gds (mS) & 0 & 1.1 & 0 & 0.70 & 0 & 3.8 \\
Tau (ps) & 0 & 5.5 & 0 & 7 & 0 & 75 \\
Rcgd (W) & 0 & 0 & 151 & 270 & 0 & 0 \\
Ri (W) & 0.3 & 1.0 & 0.01 & 0.68 & 1.0 & 1.0 \\
Rg (W) & 4.8 & 4.8 & 1.66 & 1.66 & 0.56 & 0.56 \\
Rd (W) & 1.9 & 2.7 & 1.66 & 0.94 & 2.2 & 2.2 \\
Ls (pH) & 0 & 0 & 1.1 & 1.1 & 12 & 12 \\
Ld (pH) & 32 & 32 & 49 & 49 & 60 & 60 \\
Lg (pH) & 76 & 76 & 45 & 45 & 40 & 40 \\
\hline
\end{tabular}




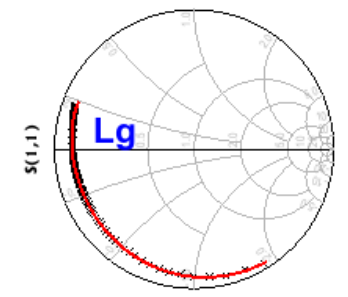

freq $(500.0 \mathrm{MHz}$ to $20.00 \mathrm{GHz})$
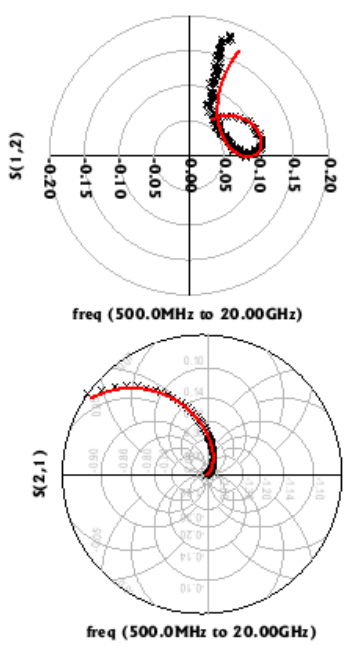
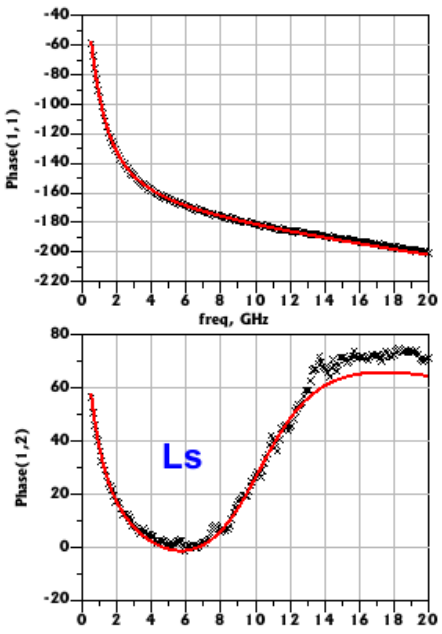
freq, $\mathbf{G H z}$

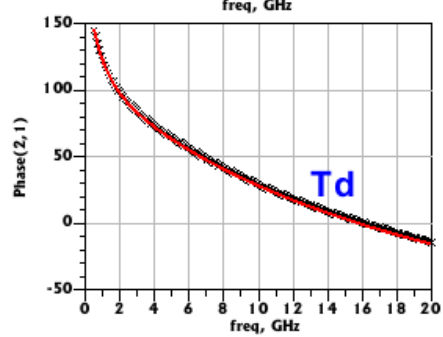

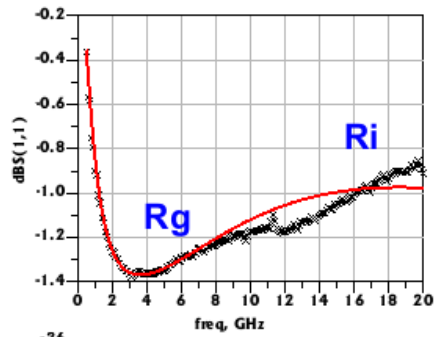

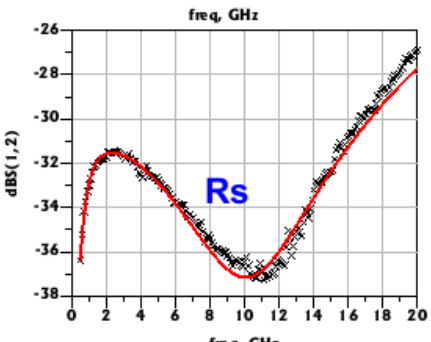
freq, GHz

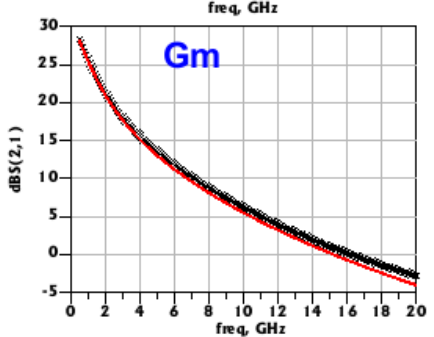

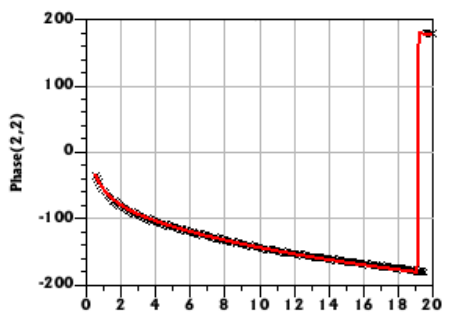

frea. $\mathbf{G H z}$
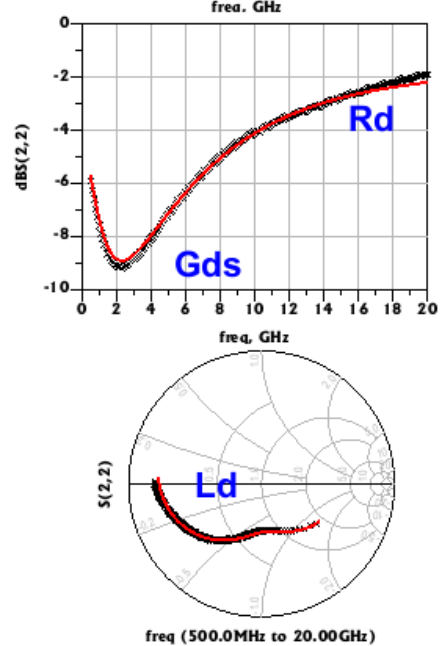

Figure 4. Modelled (continues red line) versus measured (cross black line) scattering parameters of a $4 x 400 \mu m$ structure fabricated using $0.25 \mu m$ SiC GaN technology. The regions associated to parameters extraction and/or verification are also shown.

\section{Technology Benchmarking}

High peak-to-average power ratio (PAPR) signals are needed to transfer high-volume data, driving power amplifiers to operate at high output back-off (OBO) with optimum efficiency and yet linearizable using a digital pre-distortion (DPD) system. Hence, efficiency-linearity trade-off is the most important design parameter for high power amplifier designers. From the circuit design point of view, Doherty power amplifier architecture is the industry solution for efficiency-linearity trade-off. From the technology point of view, GaN technology has the highest power density and the highest efficiency in saturation. Hence, $\mathrm{GaN}$ is the reference technology to implement highly efficient power amplifiers. The distortion generation mechanisms in GaN HEMTs devices have been investigated in various research papers i.e. [12-16]. The small-signal equivalent circuit given in Figure 3 was transformed to a simplified power amplifier model using Miller approximation in $[12,16]$; suggesting that the sources of AM/PM can be classified in two distinctive categories:

1. Technological, i.e., related to the variation of the device nonlinear parameters (essentially $\mathrm{Gm}, \mathrm{Cgd}, \mathrm{Cgs}$ and Cds).

2. Behavioral, i.e., related to the possible variation of the output conductance, GL, as for instance in the amplifier.

The nonlinearities emanating from the variation of the circuit-element with bias can be evaluated by applying a small-signal modelling technique over bias.

The presented small-signal technique is further used to evaluate these nonlinearities and to benchmark technologies. Three structures with same gate periphery from three commercially available GaN vendors were used. A multi-bias scattering parameters extraction was carried out on the three structures. Figure 5 shows $\mathrm{Cds}, \mathrm{Cgs} \mathrm{Cgd}$ and $\mathrm{Gm}$ variations versus bias for the three vendors. For sake of space, only these four parameters are shown in Figure 5. The variation of these parameters with bias is directly related to the raw static linearity or distortion of the considered process or $\mathrm{GaN}$ technology. The four parameters show the strongest voltage dependence for vendor $B$ and they show the least voltage dependence for vendor A. Another figure of merit for linearity is the ratio of on-state capacitance over the off-state capacitance. The ideal number for this ratio is 1 . In Figure 5, these ratios are:

1. Cds is $1.04,1$ and 0.95 for vendors $\mathrm{A}, \mathrm{B}$ and $\mathrm{C}$, respectively.

2. Cgs is $1.04,2.3$ and 1.5 for vendors $\mathrm{A}, \mathrm{B}$ and $\mathrm{C}$, respectively.

3. Cgd is $1,0.5$ and 1.0 for vendors $\mathrm{A}, \mathrm{B}$ and $\mathrm{C}$, respectively.

The linearity ratio is best for vendor A and worst for vendor B. 

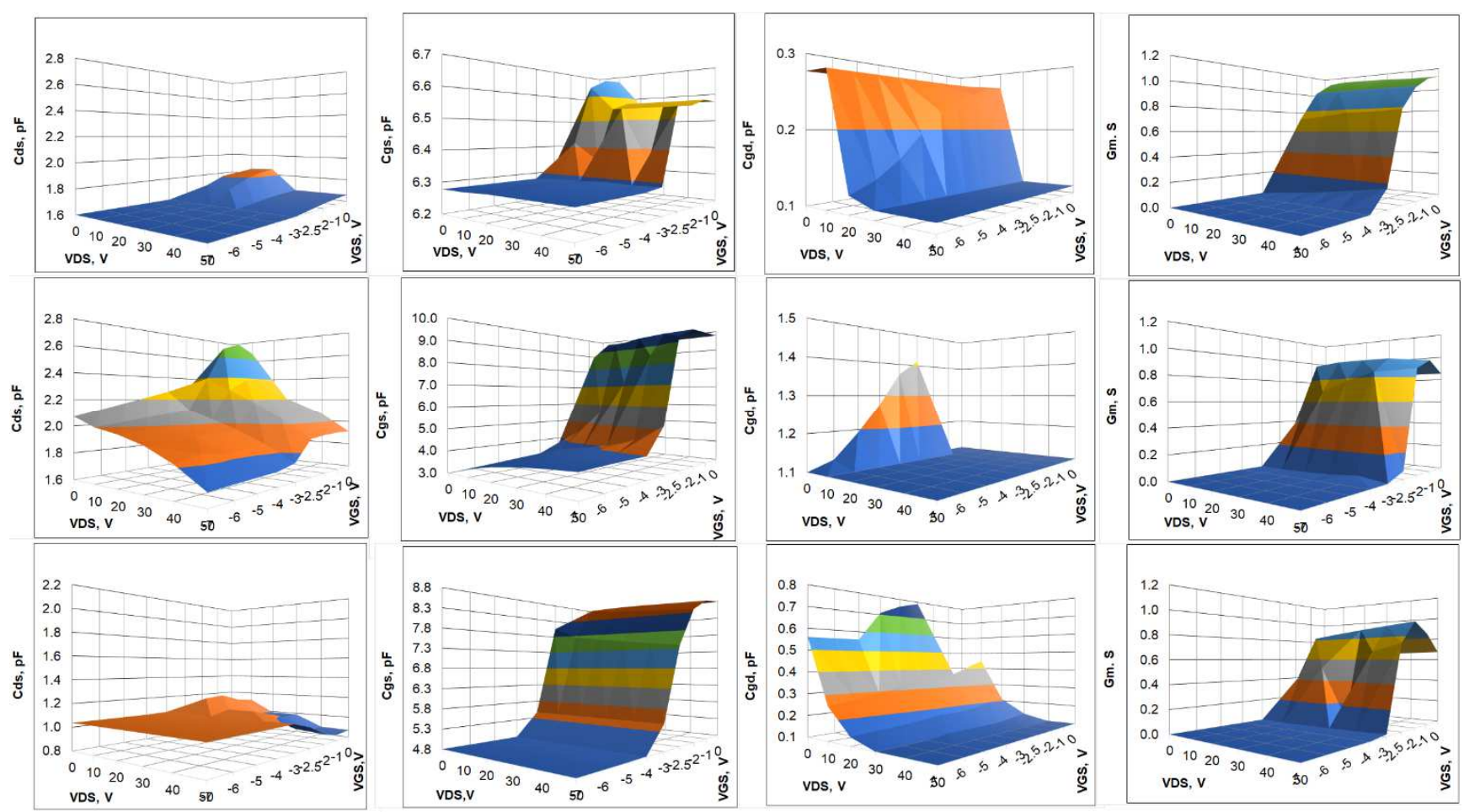

Figure 5. Cds, Cgs, Cgd and Gm variations versus bias for the three 0.5um silicon carbide GaN technology vendors. Top, middle, and bottom rows represent vendors $A, B$ and $C$ respectively.

For efficiency, smaller Cds and series resistances will result in higher efficiency. Hence, under the same design considerations, devices built with technology vendor A will show the best linearity and with that of vendor $\mathrm{C}$ will show the highest efficiency. Higher Rg and Ls will induce lower gain.

\section{Stability Analysis}

Firstly, the stability analysis tool is developed for a known stable structure, which serves as a calibration structure. Ideally, the calibration structure will consist of one or two fingers. A basic cell such as the one shown in Figure 1 can also be used. The approach consists of 4 steps:

1. Small-signal development,

2. Electromagnetic (EM) simulation,

3. Small-signal EM co-simulation,

4. Applying stability detection technique.

Here, the small-signal equivalent circuit proposed in section 2 , is developed per finger as a 1-finger small-signal model. The extraction procedure of section 2 is applied. The accuracy shown in Figure 4 is maintained.

The Graphic Design System (GDS) of the calibration structure is analyzed using an electromagnetic (EM) simulator. Only one port per finger is used as detailed in Figure 6. In general, the drain and source ports are located on the top metal layer and the gate one is located on the gate definition layer. These three coupled ports symbolize one finger for the small-signal equivalent. The substrate layer mapping will consist of all process layers, to generate an accurate EM simulation of interconnect coupling without short-circuiting the ports.
The combined 1-finger small-signal model with EM model will consist of parasitic elements obtained from EM simulation and a fraction (semiconductor part) of the core equivalent elements shown in Figure 3. It is important that the combined small-signal model simulation results maintain a good fit for the scattering and admittance parameters of the calibration structure. At this point of analysis, the tool creates an interaction between the small-signal model and EM simulation.

Secondly, to analyze a new structure such as the complex power bar shown in Figure 7, only an EM simulation of the new structure is needed to be developed and to be coupled to the small-signal model, which remains unchanged during the full analysis.

Finally, the input transfer function needed by the stability detection technique is generated. In [7], three techniques named Normalized Determinant Function [17] (NDF), large-signal NDF [18] and STAN [19] were used, all of them gave the same stability outcome, proving the accuracy of the mathematical formulation for the stability issue. The NDF function is the easiest technique to implement in the circuit simulator without any additional license and it is very easy to read its outcome. When the NDF function is associated to the present approach, it can detect odd-mode and even-mode oscillations of a given structure even before its fabrication. Figure 8 shows the NDF polar plot resulting from the analysis of the power bar given in Figure 7. The left side is the NDF function per cell and the right side is the NDF function of the total power bar structure. Knowing that from NDF stability analysis; there will be oscillation if the NDF function encircles the axis origin in clockwise direction. Figure 7 is telling us 
that the individual basic cells are stable, and the power bar is potentially unstable at $18.88 \mathrm{GHz}$. The power bar NDF plot has almost no margin at the axis origin. A slight variation in the process will cause instability.

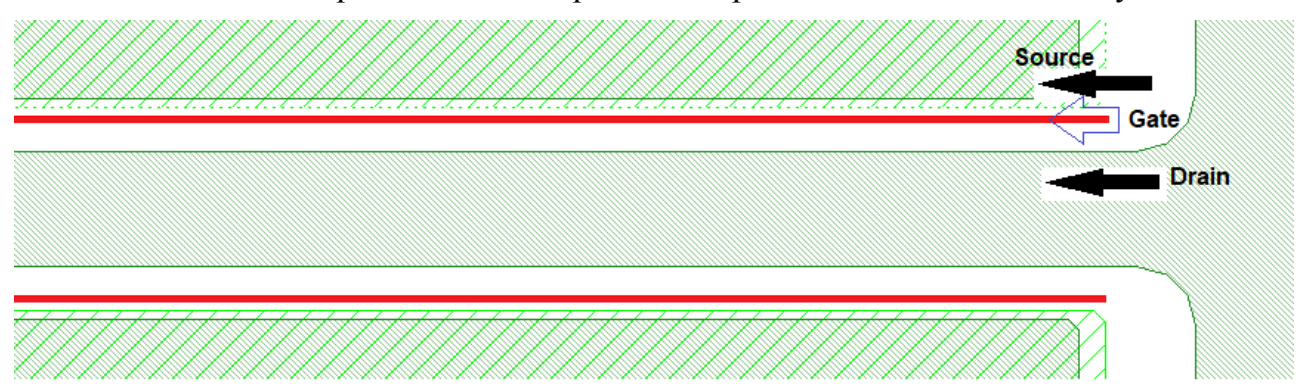

Figure 6. Port settings for EM simulation of the calibration or a structure analysis.

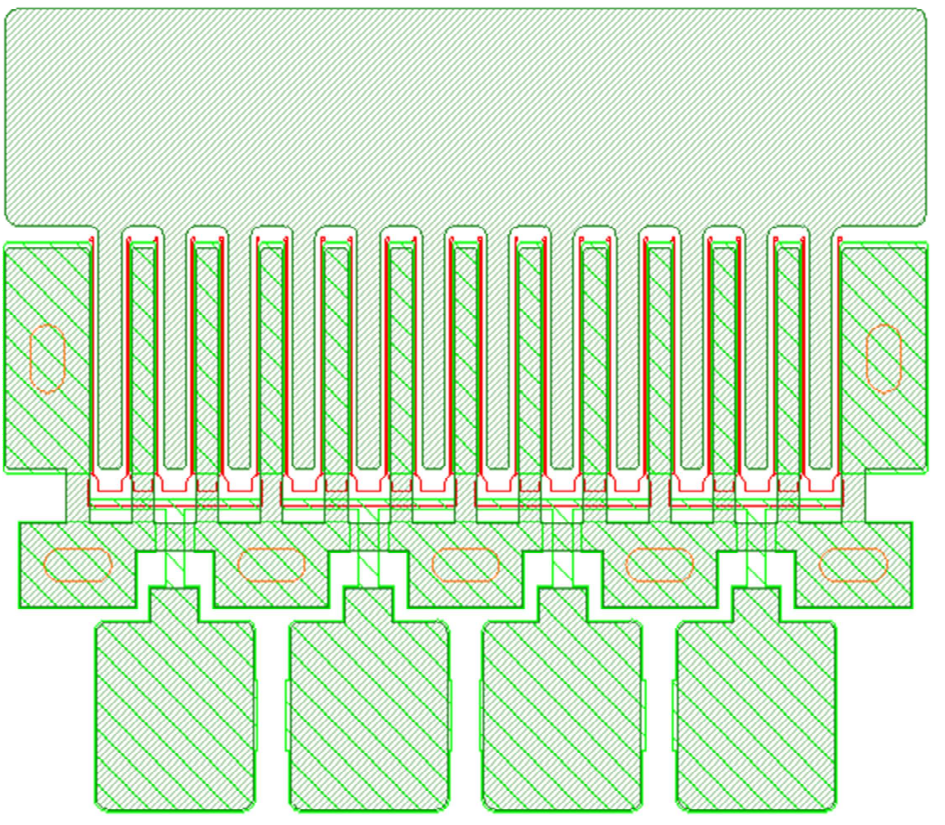

Figure 7. Power bar GaN HEMT cell with 24 fingers and 4 cells.
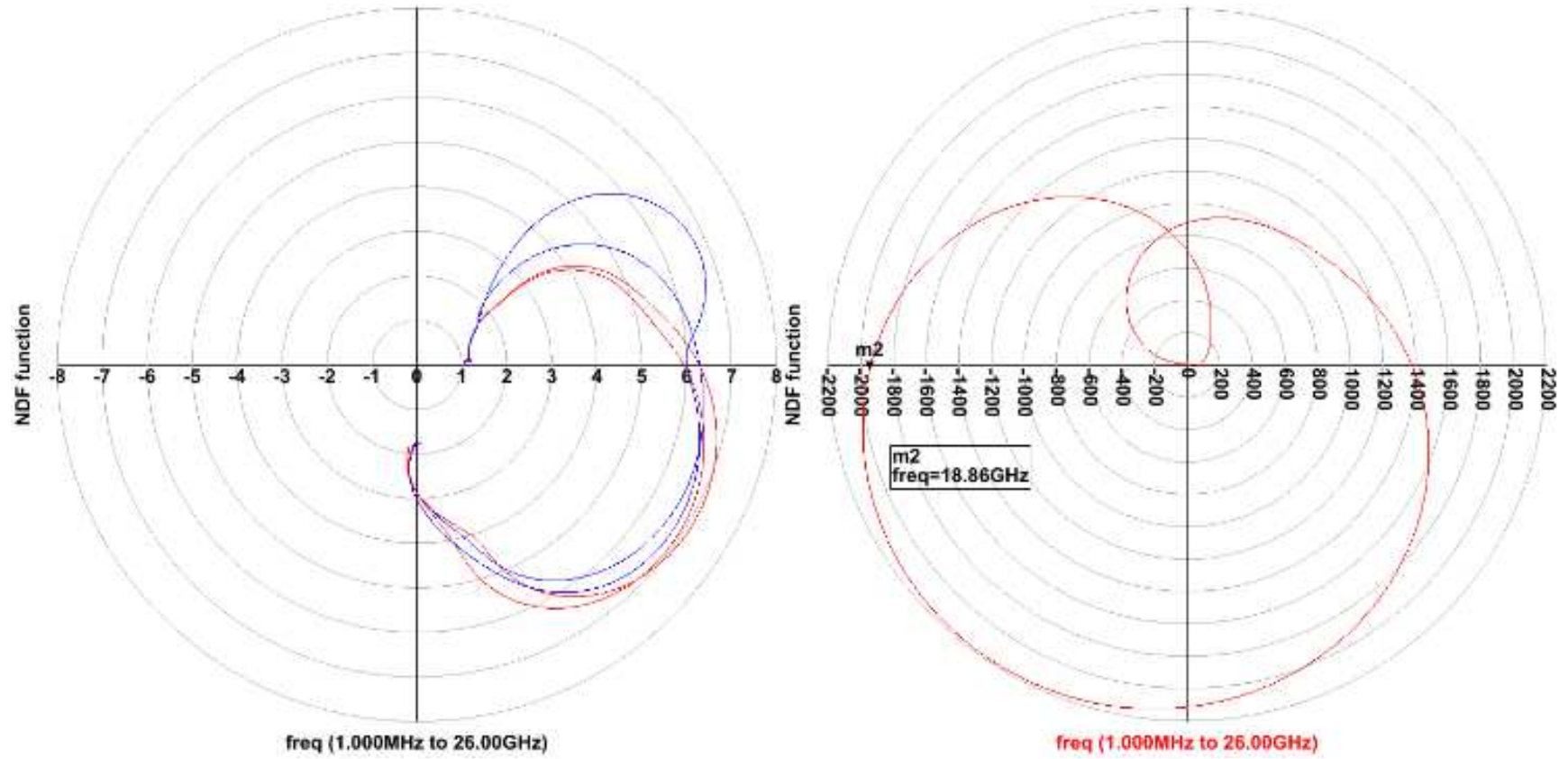

Figure 8. Polar plot of total NDF function of structure of Figure 7, showing no clockwise encirclement of the origin but a potential internal oscillation can be seen at $18.88 \mathrm{GHz}$. 
Adding a small resistor at the gate between the cells of a power bar can suppress the odd-mode oscillations [20]. A small resistor at drain side can also dump odd-mode oscillations but at the cost of efficiency points. Sometimes, this is not enough, a new way to stabilize the structure is necessary, and it is part of Intellectual Property (IP) development.

For parametric oscillation, a large-signal model is necessary to capture device non-linearities. Technology developers rely on the load pull measurements to analyze parametric oscillations of their designs.

\section{Conclusion}

The proposal discussed a practical small-signal model for GaN HEMT. Its simple semi-analytical extraction procedure was successfully applied for various technology processes and structure geometries. No unphysical parameter values were obtained even when the technique was applied for three commercial $\mathrm{GaN}$ vendors. Hence, the universality of the small-signal model and of its extraction method were demonstrated. The obtained data and the number of circuit elements were used to benchmark GaN technologies. The bias dependence of the circuit elements is related to product linearity and their absolute value to its efficiency. The proposed simple small-signal model when coupled to an electromagnetic (EM) simulation of HEMT basic cells or power bars results in an accurate stability tool analysis. The tool solves the crucial odd-mode and even-mode stability issue. The tool was demonstrated for basic cells as well as for power bar GaN HEMTs. Unquestionably, the tool can also be used for other technologies. Self-heating and trapping are common phenomena affecting GaN HEMTs, thus characterization and understanding these dynamics is critical for developing both good technology and accurate models. It would be insightful to study the evolution of the proposed small-signal model with respect to these crucial phenomena.

\section{Acknowledgements}

We would like to express our thanks and appreciation to our colleagues Dr P. Hammes, Dr. A. Dixit, and P. Jones Miet for sharing their pearls of wisdom with us during this work.

\section{References}

[1] Z. Marinković, G. Crupi, A. Caddemi, V. Marković "GaN HEMT small-signal modelling: Neural networks versus equivalent circuit," MIEL, October 2017, pp. 153-156.

[2] A. S. Hussein and A. H. Jarndal "Reliable Hybrid Small-Signal Modeling of GaN. HEMTs Based on Particle-Swarm-Optimization," IEEE Transactions on Computer-Aided Design of Integrated Circuits and Systems, Vol. 9, pp. 1816-1824, Sep. 2018.
[3] X. Du, S. K. Dhar, A. Jarndal, C Storey, M. Helaoui, S. Wingar, C. J. You, J. Cai*, F. M. Ghannouchi "Reliable Parameter Extraction of Asymmetric GaN-based Heterojunction Field Effect Transistors," EuMiC, September 2018, pp. 137-141

[4] H. Aoki, H. Sakairi, N. Kuroda, Y. Nakamura, K. Chikamatsu, K. Nakahara "A Small Signal AC Model Using Scalable Drain Current Equations of AlGaN/GaN MIS Enhancement HEMT," RFIC, June 2018, pp. 80-83.

[5] A. Khusro, M. S. Hashmi, A. Q. Ansari "Empirical Device Scaling and RF Performance Perspective: A Small Signal Model for GaN High Electron Mobility Transistor," ICNCC October 2018, pp. 45-49.

[6] G. van der Bent, A. P. de Hek, F. E. van Vliet "EM-Based GaN Transistor Small-Signal Model Scaling," EuMiC, September 2018, pp. 329-333

[7] A. Issaoun, P. Hammes, M. Fagerlind, F. Chai, T. Roedle "On Stability Analysis and Loop Oscillation of Multi-Finger GaN FET Cells for High Power Amplifiers", EuMiC, September 2018, pp. 329-333

[8] A. Jarndal, R. Essaadali, and A. B. Kouki "A reliable model parameter extraction method applied to AlGaN/GaN HEMTs," IEEE Transactions on Computer-Aided Design of Integrated Circuits and Systems, vol. 35, no. 2, pp. 211-219, Feb. 2016

[9] Y. lia, Y. Xu, Y. Wu, R. Xu, L. Zhou, T. Chen, B. Zhang “A robust small-signal equivalent circuit model for $\mathrm{AIGaN} / \mathrm{GaN}$ HEMTs up to $110 \mathrm{GHz}$," IMWS-AMP, July 2016, pp. 1-4.

[10] A. Jarndal and G. Kompa "A new small-signal modeling approach applied to GaN devices," IEEE Trans. Microwave Theory Techniques, vol. 53, no. 11, pp. 3440-3448, Nov. 2005.

[11] I. Kwon, Mi. Je, K. Lee, H. Shin "A simple and analytical parameter-extraction method of a microwave MOSFET," IEEE Trans. Microwave Theory Techniques, vol. 50, no. 6, pp. 1503-1508, June 2002.

[12] P. M. Lavrador, T. R. Cunha, P. M. Cabral, and J. C. Pedro, "The linearity-efficiency compromise," IEEE Microwave Mag., vol. 11, no. 5, pp. 44-58, August 2010.

[13] U. Radhakrishna, P. Choi, J. Grajal, L. Peh, T. Palacios, D. Antoniadis "Study of RF-circuit Linearity Performance of GAN HEMT Technology Using the MVSG Compact Device Model," IEDM, December 2016, pp. 75-78.

[14] R, Gigofré, P. Colantonio, and F. Gianinni. "A Design Approach to Maximize the Efficiency vs. Linearity Trade-Off in Fixed and Modulated Load GaN Power Amplifiers", IEEE Access, Vol. 6, 9247 - 9255, February 2018.

[15] L. C. Nunes, P. M. Cabral, J. C. Pedro, "AM/PM distortion in GaN Doherty power amplifiers", IEEE MTT-S, June 2014, pp. $1-4$

[16] V. Camarchia et al. "A Design Strategy for AM/PM Compensation in GaN Doherty Power Amplifiers." IEEE Access, Vol. 6, 22244 - 22251, November 2017.

[17] W. Struble and A. Plazker, "A Rigorous Yet Simple Method for Determining Stability of Linear N-port Networks," IEEE GaAs IC Symp. Dig., pp. 251-254, 1993. 
[18] A. Howard, “Testing an Amplifier's Stability in the Presence of a Large Signal," 2017. [Online]. Avalable: http://edadocs.software.keysight.com/display/eesofkcad $\mathrm{s} /$ Stabilit $\mathrm{y}+$ Analysis+Under+Large+Signal+Conditions .

[19] S. Dellier, "STAN Tool Stability Analysis of Microwave Circuits", in Baltimore, IMS, June 2011.
[20] A. Anakabe, J. M. Collantes, J. Portilla, S. Mons, and A. Mallet, "Detecting and avoiding oddmode parametric oscillations in microwave power amplifiers," Int. Journal of RF and Microw. Computer-Aided Engineering, Vol. 15, No. 5, 469-478, 2005. 